\title{
Selective Impairment of Processing Task-Irrelevant Emotional Faces in Cerebral Small Vessel Disease Patients
}

\author{
Zhongyu Fan (D) $1-4, *$ \\ Yunliang Guo ${ }^{1-3, *}$ \\ Xunyao Hou ${ }^{1-3}$ \\ Renjun Lv ${ }^{5}$ \\ Shanjing Nie $\mathbb{D}^{1-3}$ \\ Song $\mathrm{Xu}^{\mathrm{I}-3}$ \\ Jian Chen ${ }^{1-3}$ \\ Yan Hong ${ }^{1-3}$ \\ Shuo Zhao 6 \\ Xueping Liu ${ }^{1-3}$
}

'Department of Geriatrics, Shandong Provincial Hospital Affiliated to Shandong First Medical University, Jinan, Shandong, People's Republic of China; ${ }^{2}$ Department of Geriatric Neurology, Shandong Provincial Hospital Affiliated to Shandong First Medical University, Jinan, Shandong, People's Republic of China; ${ }^{3}$ Anti-Aging Monitoring Laboratory, Shandong Provincial Hospital Affiliated to Shandong First Medical University, Jinan, Shandong, People's Republic of China; ${ }^{4}$ Shandong Provincial Hospital, Shandong First Medical University \& Shandong Academy of Medical Sciences, Jinan, Shandong, People's Republic of China; ${ }^{5}$ The First School of Clinical Medicine, Lanzhou University, Lanzhou, Gansu, People's Republic of China; ${ }^{6}$ Department of Radiology, Shandong Provincial Hospital Affiliated to Shandong First Medical University, Jinan, Shandong, People's Republic of China

*These authors contributed equally to this work

Correspondence: Xueping Liu Department of Geriatric Neurology, Shandong Provincial Hospital Affiliated to Shandong First Medical University, Jinan, Shandong, People's Republic of China Email Liuxueping1962@163.com
Background: Few reports have implied electrophysiological alterations and neurocognitive abnormalities in patients with cerebral small vessel disease (CSVD), while no investigation is available regarding emotional processing. In the present study, pre-attentive processing of facial expressions was compared between CSVD sufferers and healthy controls using expression-related visual mismatch negativity (EMMN) as the indicator.

Methods: A total of 22 CSVD patients (12 males) and 21 age-matched healthy controls (12 males) were recruited for neuropsychological and emotional assessments, as well as electroencephalogram recording and analysis. We employed an expression-related oddball paradigm to investigate automatic emotional processing, and a series of schematic emotional faces (neutral, happy, sad) unrelated to subject's task were present in the test to avoid low-level processing of facial features.

Results: Although the distinctions of neuropsychological (MoCA and MMSE), emotional (GAD-7 and PHQ-9) and behavioral parameters (reaction time to target stimuli and response accuracy) did not reach significant levels, mean amplitudes of sad EMMN in time intervals of 150-250 ms and 250-350 ms were remarkably reduced in CSVD patients compared with healthy controls, but not for happy EMMN. Furthermore, in the control group, sad EMMN was demonstrated to be larger (more negative) than happy EMMN, while this interesting phenomenon disappeared in the CSVD group.

Conclusion: Our findings confirmed selective impairment of processing expressions which were task-irrelevant in CSVD patients, without the existence of negative bias (sad superiority) effect. The efficacy of EMMN as an electrophysiological evaluation marker of CSVD should be taken into account in future investigations.

Keywords: cerebral small vessel disease, expression-related visual mismatch negativity, preattentive processing of facial expressions, negative bias effect

\section{Introduction}

Cerebral small vessel disease (CSVD) refers to a spectrum of disorders in which pathological alterations mainly involve small blood vessels in the brain, including small arteries, arterioles, venules and capillaries. ${ }^{1}$ Its diagnosis predominantly depends on magnetic resonance imaging (MRI), with recent small subcortical infarcts, lacunes, white matter hyperintensities, microbleeds, enlarged perivascular spaces and brain atrophy considered as typical manifestations. ${ }^{2}$ Causing about $20 \%$ of strokes worldwide, CSVD is an important precipitant of cognitive impairment, including abnormalities of attention, executive function and information processing 
speed, which eventually develop into vascular dementia. ${ }^{3}$ Nevertheless, insufficient attention has been paid to early diagnosis and intervention of cognitive dysfunction for CSVD patients in clinical practice. Subjective complaints and neuropsychological assessment scales, such as Minimental State Examination (MMSE) and Montreal Cognitive Assessment (MoCA), are commonly used in cognitive evaluation and cohort study, ${ }^{4,5}$ while several subdomains (eg, emotional processing) cannot be detected by these tests, leading to rather limited sensitivity and specificity. Up to date, various attractive electrophysiological techniques are emerging, among which event-related potential (ERP) has been increasingly applied in neurological diseases, ${ }^{6,7}$ whereas few reports are available regarding CSVD. ${ }^{8}$ ERP is a specific evoked potential that reflects electrical activity of neurocognitive processing and function of specific brain regions. Owing to advantages of objectivity, noninvasiveness and high spatiotemporal resolution, it can be used to explore visual spatial attention, emotional information processing and other cognitive domains, ${ }^{9}$ which is appropriate for cognitive assessment in CSVD sufferers.

Emotional processing is a high-level cognitive procedure, which plays pivotal roles in interpersonal communication. Regarded as fundamental and necessary emotional stimuli, facial expressions transmit social information and individual's mood and are generally processed at pre-attentive stage. ${ }^{10}$ However, there is no investigation considering processing of emotional information in CSVD patients, which needs to be further illuminated.

Mismatch negativity (MMN), an extensively studied ERP component, is considered to be elicited when deviant (infrequent) stimuli are inserted in a series of standard (frequent) stimuli and violations of sequential repetition occur. Therefore, this endogenous component reflects differences between deviant and standard stimuli, and is employed as an electrophysiological index for automatic change detection and pre-attentive processing of surrounding information. ${ }^{11}$ In addition to well-documented auditory MMN, a piece of evidence has confirmed existence of its visual analogue using corresponding modalities, ${ }^{12,13}$ which can be obtained by visual physical stimuli, such as color $^{14}$ and orientation. ${ }^{15}$

Moreover, biologically relevant facial expressions also elicited visual MMN. ${ }^{16-19}$ Zhao and Li found a rightposterior expression-related visual MMN (EMMN) via an oddball paradigm of task-irrelevant emotional faces, and amplitude is observed larger for sad expressions compared with happy expressions. ${ }^{16}$ In another research, EMMN component was obtained by the usage of nonoddball sequence, with fearful and happy facial expressions served as stimuli. ${ }^{17}$ Instead of single models, additional studies indicated more reliable EMMN employing various facial models. ${ }^{18,19}$ Thus, EMMN is thought to reflect automatic processing of emotional information, and this reliable biomarker has been applied to investigate neurological and psychiatric disorders, for instance, migraine $^{20}$ and depression. ${ }^{19}$

To the best of our knowledge, the possibility of impairment in pre-attentive emotional processing for CSVD has not been verified, which will be investigated in this study. Using an oddball paradigm of schematic faces containing neutral, happy and sad expressions, we aimed to evaluate automatic processing of emotional information in CSVD sufferers by EMMN recording, measurement and analysis, together with exploring potential correlations between electrophysiological data and clinical variables. We hypothesized that CSVD patients suffered from dysfunction in processing facial expressions at pre-attentive stage.

\section{Materials and Methods Subjects and Criteria}

In this study, a total of 30 inpatients and outpatients with CSVD (16 males) were recruited from Shandong Provincial Hospital Affiliated to Shandong First Medical University, and 28 healthy controls (14 males) without systemic and neurological disorders were recruited from the local community and hospital staff. All participants underwent necessary physical examination, magnetic resonance imaging (MRI) and standardized questionnaire, then demographic and clinical characteristics of patients were collected and analyzed, such as age, gender, education, body mass index (BMI), hypertension, diabetes mellitus and hyperlipidemia.

For CSVD patients, the inclusion criteria were: 1) typical imaging features on MRI, including lacunar cerebral infarction, white matter hyperintensity, microbleed, dilated perivascular space and brain atrophy, which met the diagnostic criteria of CSVD proposed by Wardlaw et $\mathrm{al}^{2} 2$ ) aged 18 years or above; 3) clear consciousness, stable condition and satisfying cooperation throughout the test. The exclusion criteria were: 1) intracranial hemorrhage; 2) acute or history of ischemic infarction with diameter $>$ $15 \mathrm{~mm}$ or cardiogenic infarction; 3) cerebral or coronary macrovascular stenosis, such as carotid artery stenosis $(>$ 
$75 \%$; 4) abnormal findings on brain morphology caused by cerebral trauma, CO poisoning or other disorders; 5) comorbid with other neurological diseases, such as Alzheimer's disease and epilepsy; 6) suffering from systemic disorders, such as cancer and hyperthyroidism; 7) poor vision, hearing or illiterate; 8) cognitive impairment or dementia caused by anxiety, depression or other diseases. In addition, all participants were verified to be righthanded and had no significant motor or sensory dysfunction.

Furthermore 8 CSVD patients (4 males) were excluded - three for excessive electromyographic and blink interferences caused by poor cooperation, three for technical problems during recording and two for incomplete or missing clinical data. So 22 patients with CSVD (12 males) were eventually included. In terms of controls, 7 subjects ( 2 males) had to be excluded. Three for lack of neuropsychological or emotional assessments and four for conspicuous blink and drift artifacts within electroencephalogram (EEG) data. Therefore, we included a total of 21 healthy controls (12 males) for further analysis.

The investigation protocol was approved by the Ethical Committee of Shandong Provincial Hospital Affiliated to Shandong First Medical University (SWYX: NO.2020232), which was conducted in conformity to the principles and guidelines set out in Declaration of Helsinki. All subjects gave their written informed consent voluntarily before commencement of the examination.

\section{Neuropsychological and Emotional Evaluations}

Regarded as the most common and standardized screening tools, the Mini-mental State Examination (MMSE) and Montreal Cognitive Assessment (MoCA) scales were employed by specialized neuropsychologists to assess cognitive function of subjects, ${ }^{21,22}$ including multi-domain (attention, language, memory, execution, calculation, orientation and visuospatial function) and general cognition. In our investigation, these two examinations were performed on the same day with an interval of at least 3 $\mathrm{h}$, and responses were summed to obtain total scores, with MMSE $<27$ and/or MoCA $<26$ considered to be cognitive impairment.

Emotional state was evaluated with The Generalized Anxiety Disorder-7 (GAD-7) and Patient Health Questionnaire-9 (PHQ-9) scales as described in previous literatures. $^{23,24}$ Briefly, all participants were invited to respond to 7 and 9 items of questions in accordance with their experience during the last two weeks, and responses were rated on a four-point scale, ranging from 0 ("never") to 3 ("almost every day"). The total scores of aforementioned scales were summed and calculated, then cutoff scores were both set at 4 to detect anxiety and depression in CSVD patients, respectively.

\section{Stimuli, Paradigm and Experimental Procedure}

As demonstrated in Figures 1, 54 schematic faces with neutral, happy and sad expressions were presented in a computer screen (23 inches). For the purpose of avoiding low-level processing of facial features, each type of expression comprised 18 different facial models, which were displayed by adjusting the distance between facial features and the shape of facial features, especially the mouths, ${ }^{20}$ The durations of each stimulus and interstimulus interval (offset-to-onset) lasted for $150 \mathrm{~ms}$ and $450 \mathrm{~ms}$ respectively, while the visual angle was set at $3.8^{\circ}$ $\times 4.0^{\circ}$ with a viewing distance of $70 \mathrm{~cm}$.

The expression-related oddball paradigm (Figure 2) was applied in this experiment. Distinct schematic facial expressions appeared on the left and right sides of a fixation cross ("+") throughout the examination, of which neutral faces were used as standard stimuli, whereas happy and sad faces acted as deviant stimuli. This paradigm was composed of three separate sequences, each containing 211 stimuli in total (standard: 151 neutral faces, $p=0.72$; deviant: 30 happy and $30 \mathrm{sad}$ faces, both $p=0.14$ ) and was displayed in a pseudorandom order, with 10 neutral faces appearing at the beginning of each sequence and no less than two standards between consecutive deviants. The target stimuli were defined as enlarged fixation crosses ("+") presented in the central visual field, which were completely irrelevant to alteration in facial expressions.

All subjects sat comfortably in an armchair in an electrically shielded and quiet experimental chamber, and were instructed to keep their eyes on a fixation cross in the center of monitor placed in front of them. Before formal test, they were trained via three practice runs and were informed to minimize blink and body movements. In this task, participants were directed to press a button ("/") as quickly and accurately as possible when observing crosses with larger sizes, while ignoring facial stimuli in the periphery of monitor. The response accuracy and reaction time were recorded and analyzed. 


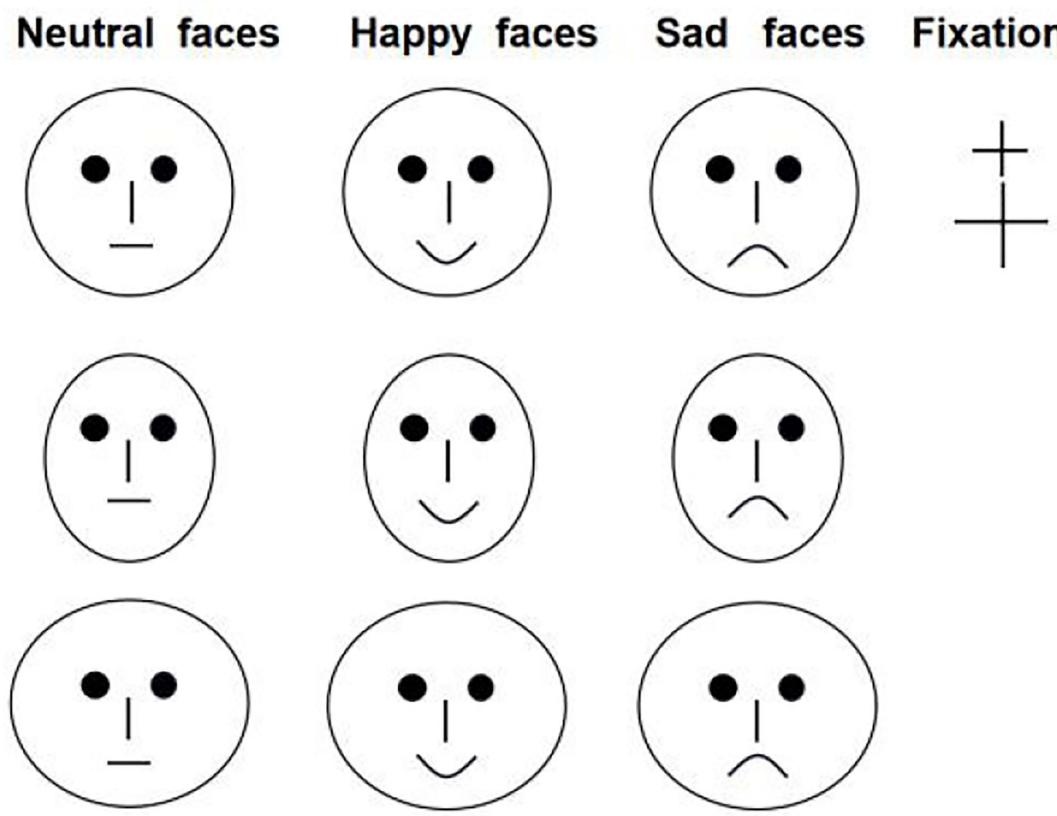

Figure I Schematic illustrations of stimuli. Examples of distinct standard stimuli (neutral faces) and deviant stimuli (happy and sad faces) mediated by changing the distance between facial features and the shape of facial features, as well as fixation crosses.

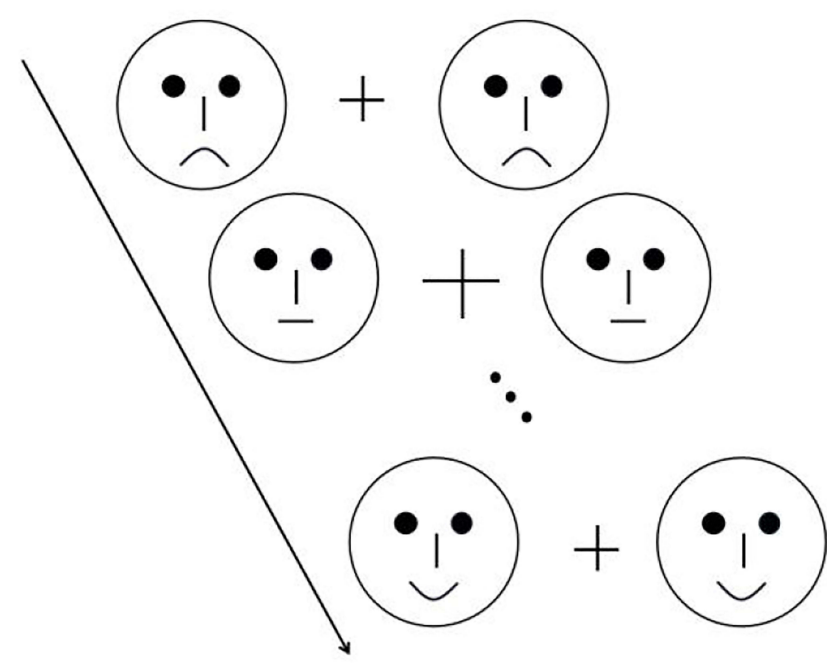

Figure 2 Schematic illustration of expression-related oddball paradigm. Each stimulus was displayed for $150 \mathrm{~ms}$, and the inter-stimulus interval was fixed at $450 \mathrm{~ms}$.

\section{Recording and Analysis of EEG Signals}

EEG signals of participants were continuously recorded with $\mathrm{Ag}-\mathrm{AgCl}$ active electrodes placed based on the 10-20 international system using Neurolab EEG/ERPs 32 Channel Amplifier (ANT Neuro, Netherland). The sampling rate, hardware high pass filter and low pass filter of recording were set at $1000 \mathrm{~Hz}, 0.05 \mathrm{~Hz}$ and $100 \mathrm{~Hz}$, respectively, and all signals were referenced to Fpz electrode. Additionally, eye movements and electrooculogram (EOG) were recorded via two pairs of electrodes placed above and below the right eye and $10 \mathrm{~mm}$ from the outer canthi. Electrode impedance was kept below $5 \mathrm{k} \Omega$ throughout the experiment.

We employed ASA 4.9.3 software to analyze EEG data. EOG artefacts were removed using independent component analysis (ICA) method, ${ }^{25}$ and digital filtering was performed with a $0.1-30 \mathrm{~Hz}(24 \mathrm{~dB} /$ octave) bandpass filter. Afterwards, EEG signals were segmented into a $600 \mathrm{~ms}$ epoch-from $100 \mathrm{~ms}$ pre-stimulus to 500 ms post-stimulus, and baseline corrections were conducted at $100 \mathrm{~ms}$ interval prior to stimuli. Segments contaminated with target stimulus (unpredictable enlargement of the fixation cross) responses, electromyographic artefacts or peak-to-peak deflection more than $\pm 100 \mu \mathrm{V}$ amplitude at any electrode were excluded from averaging. EEG segments of standard and deviant stimuli were averaged separately, and group-averaged waveforms were generated by single-subject waveforms for further analysis.

As shown in Figure 3, all facial stimuli elicited wellmarked P1 and N170 components in both groups. Moreover, two types of EMMN components, ie happy EMMN (happy minus neutral) and sad EMMN (sad minus neutral), were obtained by subtracting ERPs in response to standard stimuli (neutral faces) from those in response to deviant stimuli (happy and sad faces), respectively (see Figure 4). The mean amplitudes of EMMN 


\section{CSVD Patients}
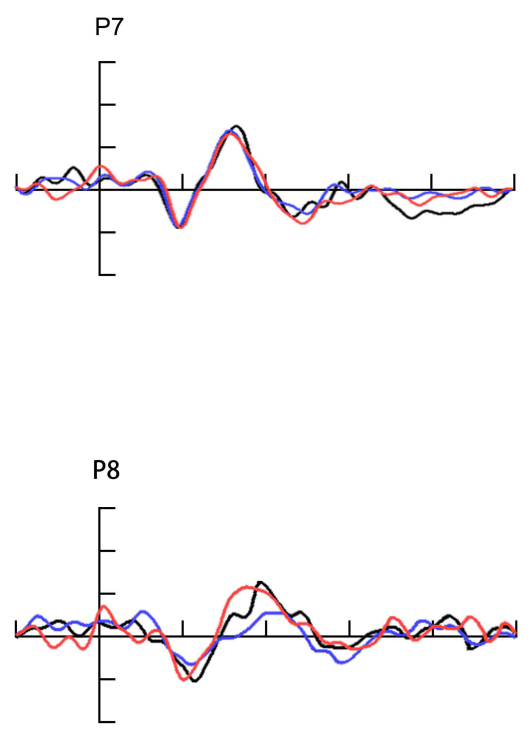

\section{Healthy Controls}

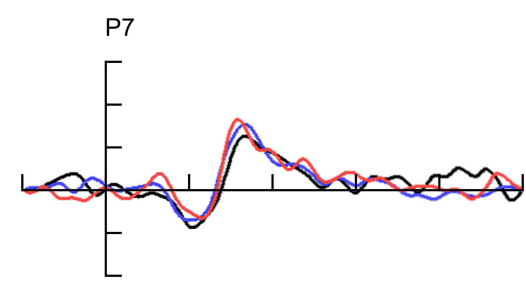

8

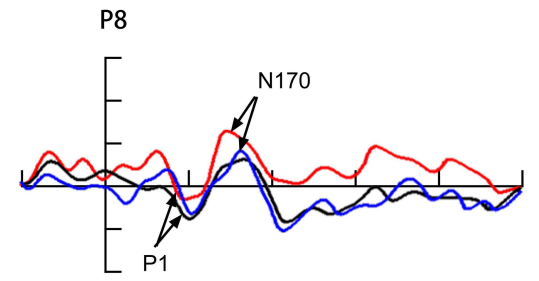

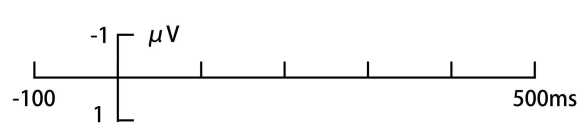

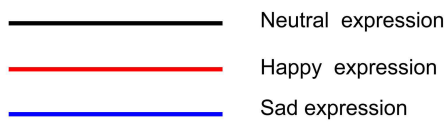

Figure 3 The grand-averaged ERP waveforms at P7/P8 sites for neutral (black), happy (blue) and sad expressions (red line) in CSVD patients and healthy controls, respectively.

were measured within two time windows-150-250 $\mathrm{ms}$ as early EMMN and 250-350 ms as late EMMN. In line with our previous investigation ${ }^{20}$ and limited by the 32 -site montage, only subset of electrode sites were used to analyze EMMN amplitudes, including lateral sites $\mathrm{P} 7$ and $\mathrm{P} 8$, more medially at $\mathrm{O} 1$ and $\mathrm{O} 2$, and left (M1) and right mastoids (M2).

During the procedure of EEG data preprocessing and measurements, researchers were blind regarding identity and diagnosis of subjects, while not for recording of EEG signals.

\section{Statistical Analysis}

All statistical analyses were performed with SPSS 26.0 (SPSS Inc., Chicago, IL, USA). Quantitative data were presented as mean \pm standard deviation (SD). Student's $t$-test for independent samples or $\chi 2$ test was employed to compare distinctions in continuous demographic, clinical, neuropsychological and emotional features between groups. As for mean EMMN amplitudes, their normal distributions were verified using Shapiro-Wilk test, followed by further analyses with repeated-measures analysis of variance (ANOVA), with expression (happy and sad), hemisphere (left and right) and electrode (P7/P8, $\mathrm{O} 1 / \mathrm{O} 2$ and M1/M2) as within-subject factors, while with group (CSVD patients vs healthy controls) as a betweensubject factor. Because of violation of sphericity hypothesis, the degrees of freedom were modified by Greenhouse-Geisser epsilon if necessary. Moreover, Bonferroni correction was conducted for post-hoc analysis of significant results. Paired $t$-test was applied for determination of statistical difference between EMMN amplitudes and zero as proposed by Kimura et al. ${ }^{17}$ Results with $P<0.05$ were considered to be statistically significant, and their effect sizes were reported as partial eta squared $(\eta 2)$. 
Fan et al

Dovepress

CSVD Patients

P7

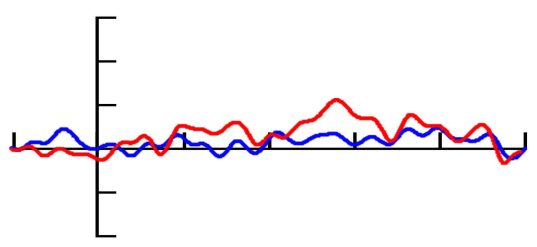

Po

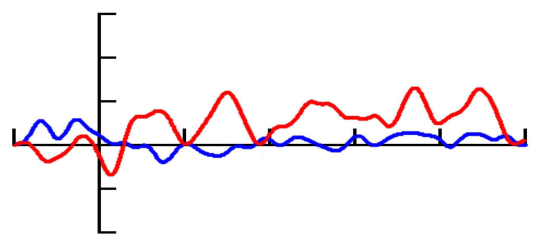

01

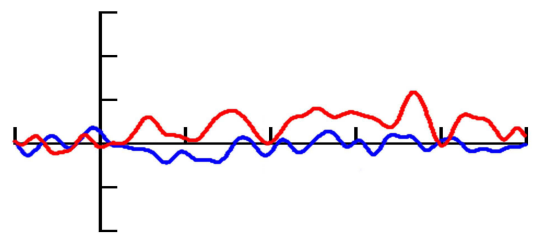

02
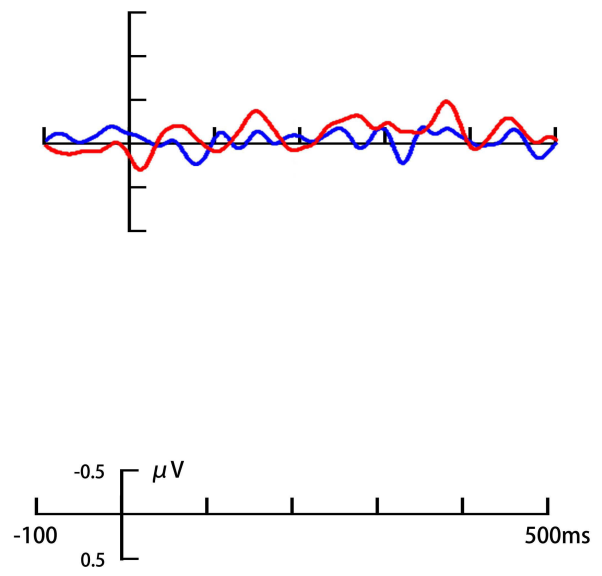

Healthy Controls

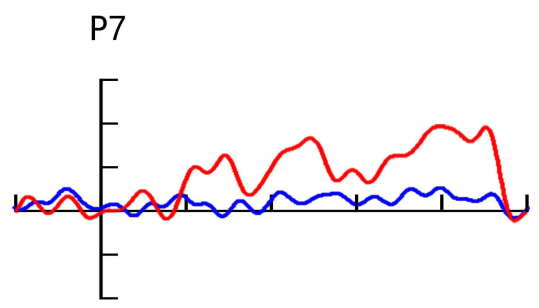

Po

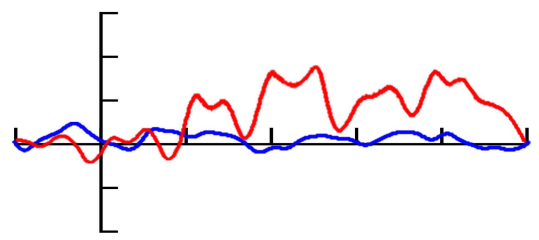

01

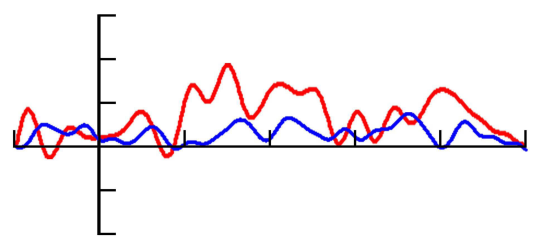

02

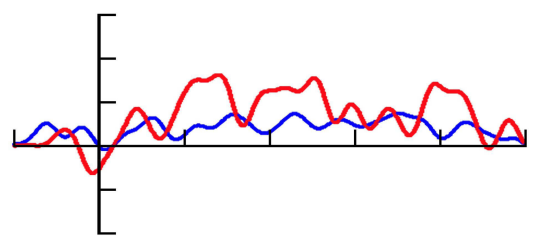

Happy EMMN

Sad EMMN

Figure 4 The grand-averaged EMMN waveforms at P7/P8 and OI/O2 sites in CSVD patients and healthy controls, respectively. Blue line indicates happy EMMN (happy minus neutral) and red line sad EMMN (sad minus neutral).

Results

Sample Characteristics

The demographic and clinical characteristics of CSVD patients and healthy controls were demonstrated in Table 1. No notable difference was observed in age, gender, education and BMI parameter between two groups (all $p>0.05)$. As for neuropsychological and emotional features, evaluated by MoCA/MMSE and GAD-7/PHQ9 , respectively, the corresponding analyses did not reach significant levels either $(p s>0.05)$.

Behavioral Performance

The reaction time to target stimuli and response accuracy were recorded and analyzed. Neither reaction time (CSVD

3698

https://doi.org/10.2147/NDT.S340680

Neuropsychiatric Disease and Treatment 2021:17

Dove Press 
Table I Sample Characteristics

\begin{tabular}{|c|c|c|c|c|}
\hline & CSVD Patients & Healthy Controls & $t / \chi^{2}$ & $\boldsymbol{P}$ \\
\hline Number & 22 & 21 & & \\
\hline Age, years & $60.05 \pm 9.64$ & $56.76 \pm 10.31$ & 1.08 & 0.287 \\
\hline Age range, years & $37-72$ & $36-70$ & & \\
\hline Gender, male/female & $12 / 10$ & $12 / 9$ & 1.00 & 0.554 \\
\hline Education, years & $13.14 \pm 1.73$ & $14.05 \pm 1.69$ & -1.75 & 0.088 \\
\hline $\mathrm{BMI}\left(\mathrm{kg} / \mathrm{m}^{2}\right)$ & $25.53 \pm 2.43$ & $24.79 \pm 3.04$ & 0.88 & 0.383 \\
\hline With hypertension number (percentage) & 12 (54.55\%) & - & - & - \\
\hline With diabetes number (percentage) & $10(45.45 \%)$ & - & - & - \\
\hline With hyperlipidemia number (percentage) & $15(68.18 \%)$ & - & - & - \\
\hline MoCA & $26.91 \pm 2.07$ & $27.90 \pm 1.73$ & $-1.7 \mid$ & 0.095 \\
\hline MMSE & $26.95 \pm 1.73$ & $27.76 \pm 1.04$ & -1.84 & 0.073 \\
\hline GAD-7 & $2.86 \pm 1.36$ & $2.29 \pm 1.15$ & 1.51 & 0.140 \\
\hline PHQ-9 & $2.50 \pm 1.10$ & $2.00 \pm|.4|$ & 1.30 & 0.202 \\
\hline
\end{tabular}

Notes: Data were expressed as mean \pm SD (Standard Deviation). Student's $t$-test was used in analyses of age, education, BMI, MoCA, MMSE, GAD-7 and PHQ-9. $\chi^{2}$ test was used in analysis of gender.

Abbreviations: BMI, body mass index; MoCA, Montreal Cognitive Assessment; MMSE, Mini-mental State Examination; GAD-7, The Generalized Anxiety Disorder-7; PHQ9, The Patient Health Questionnaire-9; $\chi^{2}$, chi-square.

patients, $370.5 \pm 37.0 \mathrm{~ms}$; healthy controls, $361.0 \pm 42.1$ $\mathrm{ms} ; t=0.78, p=0.438$ ) nor response accuracy (CSVD patients, $95.0 \pm 2.7 \%$; healthy controls, $95.7 \pm 2.4 \%, t=-$ $0.77, p=0.443)$ were statistically significant.

\section{EMMN Analysis}

Figure 3 shows grand-averaged ERP waveforms elicited by facial expressions (neutral, happy and sad) in CSVD patients and healthy controls at P7/P8 sites, respectively. The grand-averaged waveforms of subtraction-derived EMMN (happy EMMN and sad EMMN) are displayed in Figure 4, and mean EMMN amplitudes within the ranges of $150-250 \mathrm{~ms}$ and $250-350 \mathrm{~ms}$ were analyzed (Table 2).

As for early EMMN (150-250 ms), mean amplitude was significantly attenuated in CSVD patients $(-0.08 \pm$ $0.91 \mu \mathrm{V})$ compared with healthy controls $(-0.47 \pm 0.48$ $\mu \mathrm{V}, \mathrm{F}(1,41)=8.96, p=0.005$, partial $\eta 2=0.18)$. Moreover, EMMN amplitude was modulated by facial expressions $(\mathrm{F}(1.00,41.00)=11.64, p=0.001$, partial $\eta 2$ $=0.22$ ), and the interaction of group and expression was verified to be remarkable $(\mathrm{F}(1.00,41.00)=4.45, p=$ 0.041, partial $\eta 2=0.10$ ) (Table 2). Post-hoc analyses revealed that, under sad condition, CSVD sufferers $(-0.06 \pm 0.93 \mu \mathrm{V})$ exhibited reduced amplitude than that in the control group $(-0.70 \pm 0.58 \mu \mathrm{V}, \mathrm{F}(1,41)=$ 15.08, $p<0.001$, partial $\eta 2=0.27)$, but not for happy expression (CSVD patients, $-0.10 \pm 0.85 \mu \mathrm{V}$; healthy controls, $-0.24 \pm 0.62 \mu \mathrm{V} ; \mathrm{F}(1,41)=1.67, p=0.203)$.
Additionally, in controls, the amplitude of sad EMMN was observed to be larger (more negative) in comparison with happy EMMN $(p<0.001$, partial $\eta 2=0.28)$, while this pattern was not found in patients $(p=0.368)$. We also obtained noticeable electrode effect (F $(1.99,81.72)=3.35, p=0.040$, partial $\left.\eta^{2}=0.08\right)$, of which the negative amplitude was largest at P7/P8 sites $(-0.32 \pm 0.72 \mu \mathrm{V})$.

Within the 250-350 ms window, the main effects of group (CSVD patients, $-0.11 \pm 0.62 \mu \mathrm{V}$; healthy controls, $-0.56 \pm 0.39 \mu \mathrm{V} ; \mathrm{F}(1,41)=14.68, p<0.001$, partial $\eta 2=$ $0.26)$ and ex-pression $(\mathrm{F}(1.00,41.00)=11.23, p=0.002$, partial $\eta 2=0.22)$, as well as group $\times$ expression interaction $(\mathrm{F}(1.00,41.00)=7.42, p=0.009$, partial $\eta 2=0.15)$, were all statistically significant (see Table 2). Subsequent comparisons were consistent with the abovementioned results of early EMMN. Moreover, remarkable electrode effect $(\mathrm{F}(1.80,73.89)=5.38, p=0.008$, partial $\eta 2=0.116)$ was observed, and it interacted with group $(\mathrm{F}(1.80,73.89)$ $=4.62, p=0.016$, partial $\eta 2=0.101)$, implying that patients produced smaller MMN response to facial expressions compared with their control counterparts at $\mathrm{P} 7 / \mathrm{P} 8$ $(p<0.001$, partial $\eta 2=0.40)$ and $\mathrm{O} 1 / \mathrm{O} 2$ sites $(p=0.001$, partial $\eta 2=0.25)$, but not at M1/M2 sites $(p=0.71)$. We also discovered that the interactions of expression $\times$ electrode and group $\times$ expression $\times$ electrode reached significant levels $(\mathrm{F}(1.48,60.54)=5.04, p=0.017$, partial $\eta 2=$ 0.11 and $\mathrm{F}(1.48,60.54)=5.22, p=0.015$, partial $\eta 2=0.12$, respectively). 
Table 2 Results and Analysis of Mean Amplitude $(\mu \mathrm{V})$ of EMMN in Healthy Control and Patients with CSVD

\begin{tabular}{|c|c|c|c|c|c|c|c|c|}
\hline \multirow[t]{2}{*}{ EMMN } & & \multicolumn{2}{|c|}{ CSVD Patients } & \multicolumn{2}{|c|}{ Healthy Controls } & \multicolumn{3}{|c|}{ Statistics } \\
\hline & & Happy & Sad & Happy & Sad & Group $\mathbf{F}^{\mathbf{a}}$ & Expression $\mathbf{F}^{\mathbf{b}}$ & Group $\times$ Expression $F^{c}$ \\
\hline $150-250 \mathrm{~ms}$ & Amplitude $(\mu \mathrm{V})$ & $-0.10 \pm 0.85$ & $-0.06 \pm 0.93$ & $-0.24 \pm 0.62$ & $-0.70 \pm 0.58$ & $8.96 * *$ & $11.64 * * *$ & $4.45^{*}$ \\
\hline $250-350 \mathrm{~ms}$ & Amplitude $(\mu \mathrm{V})$ & $-0.18 \pm 0.77$ & $-0.04 \pm 0.59$ & $-0.38 \pm 0.46$ & $-0.74 \pm 0.38$ & $14.68 * * *$ & $11.23 * * *$ & $7.42^{* *}$ \\
\hline
\end{tabular}

Notes: Data were expressed as mean $\pm \mathrm{SD}$. EMMN mean amplitude was measured within two time windows $(150-250 \mathrm{~ms}$ and $250-350 \mathrm{~ms})$. $* \mathrm{P}<0.05$, $* * \mathrm{P}<0.0 \mathrm{I}, * * * \mathrm{P}<$ 0.001 by repeated-measures ANOVA (Bonferroni correction). ${ }^{\mathrm{a}} \mathrm{F}$ value of main effect of group. ${ }^{\mathrm{b}} \mathrm{F}$ value of main effect of expression. ${ }^{\mathrm{c}} \mathrm{F}$ value of interaction of group and expression.

Abbreviation: EMMN, expression-related mismatch negativity.

To verify the existence of EMMN, further comparisons between EMMN amplitudes and zero were performed for two groups. In control participants, during early period (150-250 ms), mean amplitudes of happy and sad EMMN were significantly different from zero at $\mathrm{P} 7 / \mathrm{P} 8$ and $\mathrm{O} 1 / \mathrm{O} 2$ sites ( $p s<0.05$ by paired $t$-test). In terms of late period (250-350 ms), sad EMMN was remarkably elicited at each channel (P7/P8, O1/O2 and M1/M2) (ps $<0.05$ ), while for happy EMMN, the comparisons were statistically significant only at P7/P8 sites (both $p<0.01$ ). Nevertheless, in CSVD patients, there existed no observable MMN response towards emotional faces at any electrodes, regardless of happy or sad condition (all $p>0.05$ ).

\section{Discussion}

In the present study, using an expression-related oddball paradigm with neutral, happy and sad faces as stimuli, we comprehensively investigated pre-attentive processing of facial expressions for CSVD. Although no difference was observed in behavioral performance (reaction time and response accuracy) between groups, the average amplitudes of EMMN were markedly decreased in CSVD sufferers compared with healthy controls, especially under happy condition. These findings implicated that CSVD patients might suffer from impairments in automatic emotional processing, particularly towards happy expressions.

In this work, significant group effects were obtained for mean EMMN amplitudes within time windows of $150-250 \mathrm{~ms}$ and $250-350 \mathrm{~ms}$, and post-hoc analyses showed that the amplitudes of sad EMMN were dramatically attenuated in comparison with control counterparts, while not for happy faces. Since EMMN component is generally elicited in the absence of attention and reflects ability in automatic change detection of emotional information, ${ }^{16-18,26}$ the abovementioned results implied abnormalities in pre-attentive emotional processing for
CSVD patients, which selectively involved sad expressions.

Another key finding of our investigation was that in two ranges calculated, EMMN amplitudes elicited by sad faces were remarkably larger (more negative) than those elicited by happy faces in healthy controls, suggesting that control participants allocated more processing resources to sad expressions and other negative emotional information even at pre-attentive stage. It has been well documented that negative stimuli, including facial expressions, are inclined to be handled more intensely and swiftly than positive ones under attentional condition. ${ }^{27-29}$ From an electrophysiological perspective, the recording and analysis of EMMN in controls further supported the negative bias (sad superiority) theory in field of automatic processing, which corroborated observations in previous reports. ${ }^{16,17,30}$ In contrast, corresponding analyses regarding negativity bias effect did not reach significant levels in CSVD patients, demonstrating that there appeared to be no distinction between happy and sad faces without focused attention to facial expressions.

The selective impairment in processing of sad faces and disappearance of negative bias effect were discovered in CSVD patients, and these interesting phenomena might be explained by the hypothesis that perceptions of distinct facial expressions were associated with dissociable neural structures. ${ }^{31}$ A piece of evidence has indicated that thalamic nuclei, especially pulvinar nucleus, are sensitive to changes in blood supply ${ }^{32}$ and are extensively connected with cortical (sensory cortices, posterior parietal cortex, prefrontal cortex) and subcortical regions (superior colliculus, amygdala). ${ }^{33-35}$ More importantly, the pulvinar is dispensable in both multisensory sensory and fear processing, so problems with blood supply to pulvinar nucleus can lead to abnormal sensory emotional response. ${ }^{33,34,36,37}$ Previous publications demonstrated that white matter density of pulvinar-amygdala connection was significantly 
correlated with capability to recognize and process emotional faces, ${ }^{36,38}$ and white matter hyperintensity is regarded as a common sign in CSVD imaging. Thus, there is the possibility that ischemia in specific regions can cause alterations of certain neural circuits, which contributes to impairment of negative emotional processing in succession, while the network-level pathology and exact mechanisms deserve further elucidation for CSVD sufferers, perhaps by fMRI technique.

Moreover, the global cognitive function evaluated by MoCA and MMSE scales seemed to remain intact in CSVD patients. As suggested by prior publication, traditional neuropsychological assessment tools displayed different sensitivities and specificities in screening of cognitive impairment, for instance, MoCA possessed higher sensitivity ( 0.97 vs 0.65$)$ while lower specificity (0.60 vs 0.89$)$ compared with MMSE. ${ }^{39}$ Nevertheless, divergent results have been obtained for neuropsychological evaluation by other CSVD-related reports, ${ }^{5,40}$ and the inconsistencies might arise from diverse subject selection criteria and research protocols, which needs to be illuminated in future investigation using larger cohorts. Notably, mean amplitudes of sad EMMN were markedly attenuated in CSVD sufferers than those in healthy controls, implying that EMMN component could serve as a reliable biomarker to reflect dysfunction of emotional processing and ERP experiments might detect early cognitive decline sensitively for CSVD, at least indicated by this study.

To the best of our knowledge, using an expressionrelated oddball paradigm, we comprehensively investigated emotional processing in CSVD patients for the first time and revealed relevant dysfunction. Moreover, low-level processing was avoided by manipulating the distance between facial features and the shape of facial features. Standardized subject enrollment criteria and collection of detailed information were also included in strengths of this study. However, several limitations constrained the explanation of our findings. Firstly, confined with small sample size, the effects of age and gender on pre-attentive processing of facial expressions were not explored, and we aimed to further characterize correlations between clinical data (eg, imaging features) and EMMN abnormalities. Secondly, a group of schematic facial models with neural, happy and sad expressions were applied in this experiment owing to their simplicity and reliability, ${ }^{26}$ while stimuli of human real faces were still needed to reconfirm our observations. Finally, source localization of EMMN in CSVD sufferers was not performed, which should be studied in future research to uncover the underlying mechanisms.

\section{Conclusions}

Notwithstanding these limitations, in the present investigation, we revealed that although global cognitive function assessed by MoCA and MMSE remained intact, CSVD patients suffered from selective impairment in automatic processing of sad expressions in comparison with healthy controls, while not for happy expressions. Furthermore, the negative bias effect was not observed for CSVD, indicating no apparent superiority in processing of sad faces compared with happy faces at pre-attentive stage. Given this, considering high prevalence of CSVD in the elderly, the issue of neurocognitive processing abnormalities should be paid critical attention, particularly for early diagnosis and intervention. Our findings shed light on the sensitivity and specificity of ERP tests, and further studies are warranted to determine whether EMMN component can serve as a promising electrophysiological indicator for cognitive evaluation in CSVD sufferers.

\section{Institutional Review Board Statement}

The investigation protocol was approved by the Ethical Committee of Shandong Provincial Hospital Affiliated to Shandong First Medical University (SWYX: NO.2020232), which was conducted in conformity to the principles and guidelines set out in Declaration of Helsinki.

\section{Informed Consent Statement}

Informed consent was obtained from all individual participants included in the study.

\section{Author Contributions}

All authors made a significant contribution to the work reported, whether that is in the conception, study design, execution, acquisition of data, analysis and interpretation, or in all these areas; took part in drafting, revising or critically reviewing the article; gave final approval of the version to be published; have agreed on the journal to which the article has been submitted; and agree to be accountable for all aspects of the work.

\section{Funding}

This work was supported by grants from Key Research \& Development project in Shandong Province 
[No. 2019GSF108101 (X.L.)] and Foundation of Youth Talent of Shandong Provincial Hospital Affiliated to Shandong First Medical University (Y.G.).

\section{Disclosure}

The authors declare that they have no conflicts of interest.

\section{References}

1. Charidimou A, Pantoni L, Love S. The concept of sporadic cerebral small vessel disease: a road map on key definitions and current concepts. Int J Stroke. 2016;11(1):6-18. doi:10.1177/174749301 5607485

2. Wardlaw JM, Smith EE, Biessels GJ, et al. Neuroimaging standards for research into small vessel disease and its contribution to ageing and neurodegeneration. Lancet Neurol. 2013;12(8):822-838. doi:10.1016/S1474-4422(13)70124-8

3. Zanon Zotin MC, Sveikata L, Viswanathan A, et al. Cerebral small vessel disease and vascular cognitive impairment: from diagnosis to management. Curr Opin Neurol. 2021;34(2):246-257.

4. Peng D, Chen L, Chen X. Clinical practice guideline for cognitive impairment of cerebral small vessel disease. Aging Med. 2019;2 (2):64-73. doi:10.1002/agm2.12073

5. Jiang Y, Wang Y, Yuan Z, et al. Total cerebral small vessel disease burden is related to worse performance on the mini-mental state examination and incident dementia: a prospective 5-year follow-up. J Alzheimers Dis. 2019;69(1):253-262. doi:10.3233/JAD-181135

6. Guo $\mathrm{Y}, \mathrm{Xu} \mathrm{S}$, Nie $\mathrm{S}$, et al. Female versus male migraine: an event-related potential study of visual neurocognitive processing. J Headache Pain. 2019;20(1):38. doi:10.1186/s10194-019-0995-y

7. Morrison C, Rabipour S, Knoefel F, et al. Auditory event-related potentials in mild cognitive impairment and Alzheimer's disease. Curr Alzheimer Res. 2018;15(8):702-715. doi:10.2174/1567205 015666180123123209

8. Wang C, Gao J, Li M, et al. Association of cognitive impairment and mood disorder with event-related potential P300 in patients with cerebral small vessel diseases. Neuro Endocrinol Lett. 2019;40(78):333-341.

9. Helfrich RF, Knight RT. Cognitive neurophysiology: event-related potentials. Handb Clin Neurol. 2019;160:543-558.

10. Compton RJ. The interface between emotion and attention: a review of evidence from psychology and neuroscience. Behav Cogn Neurosci Rev. 2003;2(2):115-129. doi:10.1177/1534582303002002003

11. Näätänen R, Pakarinen S, Rinne T, et al. The mismatch negativity (MMN): towards the optimal paradigm. Clin Neurophysiol. 2004;115 (1):140-144. doi:10.1016/j.clinph.2003.04.001

12. Pazo-Alvarez P, Cadaveira F, Amenedo E. MMN in the visual modality: a review. Biol Psychol. 2003;63(3):199-236. doi:10.1016/ S0301-0511(03)00049-8

13. File D, Sulykos I, Czigler I. Automatic change detection and spatial attention: a visual mismatch negativity study. Eur $J$ Neurosci. 2020;52(11):4423-4431. doi:10.1111/ejn.13945

14. Czigler I, Balazs L, Winkler I. Memory-based detection of task-irrelevant visual changes. Psychophysiology. 2002;39 (6):869-873. doi:10.1111/1469-8986.3960869

15. Kimura M, Katayama J, Ohira H, et al. Visual mismatch negativity: new evidence from the equiprobable paradigm. Psychophysiology. 2009;46(2):402-409. doi:10.1111/j.1469-8986.2008.00767.x

16. Zhao L, Li J. Visual mismatch negativity elicited by facial expressions under non-attentional condition. Neurosci Lett. 2006;410 (2):126-131. doi:10.1016/j.neulet.2006.09.081

17. Kimura M, Kondo $\mathrm{H}$, Ohira $\mathrm{H}$, et al. Unintentional temporal context-based prediction of emotional faces: an electrophysiological study. Cereb Cortex. 2012;22(8):1774-1785. doi:10.1093/cercor/bhr244
18. Astikainen P, Hietanen JK. Event-related potentials to task-irrelevant changes in facial expressions. Behav Brain Funct. 2009;5:30. doi:10.1186/1744-9081-5-30

19. Chang Y, Xu J, Shi N, et al. Dysfunction of processing task-irrelevant emotional faces in major depressive disorder patients revealed by expression-related visual MMN. Neurosci Lett. 2010;472(1):33-37. doi:10.1016/j.neulet.2010.01.050

20. Guo Y, Chen J, Hou X, et al. Pre-attentive dysfunction of processing emotional faces in interictal migraine revealed by expression-related visual mismatch negativity. Brain Res. 2020;1738:146816. doi:10. 1016/j.brainres.2020.146816

21. Lim MYL, Loo JHY. Screening an elderly hearing impaired population for mild cognitive impairment using Mini-Mental State Examination (MMSE) and Montreal Cognitive Assessment (MoCA). Int $J$ Geriatr Psychiatry. 2018;33(7):972-979. doi:10. 1002/gps. 4880

22. Nasreddine ZS, Phillips NA, Bédirian V, et al. The Montreal Cognitive Assessment, MoCA: a brief screening tool for mild cognitive impairment. $J$ Am Geriatr Soc. 2005;53(4):695-699. doi:10.11 11/j.1532-5415.2005.53221.x

23. Spitzer RL, Kroenke K, Williams JBW, et al. A brief measure for assessing generalized anxiety disorder: the GAD-7. Arch Intern Med. 2006;166(10):1092-1097. doi:10.1001/archinte.166.10.1092

24. Dajpratham P, Pukrittayakamee P, Atsariyasing W, et al. The validity and reliability of the PHQ-9 in screening for post-stroke depression. BMC Psychiatry. 2020;20(1):291. doi:10.1186/s12888020-02699-6

25. Jung TP, Makeig S, Humphries C, et al. Removing electroencephalographic artifacts by blind source separation. Psychophysiology. 2000;37(2):163-178. doi:10.1111/1469-8986.3720163

26. Kreegipuu K, Kuldkepp N, Sibolt O, et al. vMMN for schematic faces: automatic detection of change in emotional expression. Front Hum Neurosci. 2013;7:714. doi:10.3389/fnhum.2013.00714

27. Baumeister RF, Bratslavsky E, Finkenauer C, et al. Bad is stronger than good. Rev Gen Psychol. 2001;5(4):323-370. doi:10.1037/10892680.5.4.323

28. Hansen $\mathrm{CH}$, Hansen RD. Finding the face in the crowd: an anger superiority effect. J Pers Soc Psychol. 1988;54(6):917-924. doi:10.1037/0022-3514.54.6.917

29. Fox E, Lester V, Russo R, et al. Facial expressions of emotion: are angry faces detected more efficiently? Cogn Emot. 2000;14(1):61-92. doi:10.1080/026999300378996

30. Stefanics G, Csukly G, Komlósi S, et al. Processing of unattended facial emotions: a visual mismatch negativity study. Neuroimage. 2012;59(3):163-178. doi:10.1016/j.neuroimage.2011.10.041

31. Atkinson AP, Adolphs R. The neuropsychology of face perception: beyond simple dissociations and functional selectivity. Philos Trans $R$ Soc Lond B Biol Sci. 2011;366(1571):61-92. doi:10.1098/rstb. 2010.0349

32. Bordes S, Werner C, Mathkour M, et al. Arterial supply of the thalamus: a comprehensive review. World Neurosurg. 2020;37: 310-318. doi:10.1016/j.wneu.2020.01.237

33. Chou XL, Fang Q, Yan L, et al. Contextual and cross-modality modulation of auditory cortical processing through pulvinar mediated suppression. Elife. 2020;9:e54157. doi:10.7554/eLife.54157

34. Fang Q, Chou X-L, Peng B, et al. A differential circuit via retino-colliculo-pulvinar pathway enhances feature selectivity in visual cortex through surround suppression. Neuron. 2020;105 (2):355-369.e6. doi:10.1016/j.neuron.2019.10.027

35. Ibrahim LA, Mesik L, Ji XY, et al. Cross-modality sharpening of visual cortical processing through layer-1-mediated inhibition and disinhibition. Neuron. 2016;89(5):1031-1045. doi:10.1016/j.neuron. 2016.01.027

36. McFadyen J, Mattingley JB, Garrido MI. An afferent white matter pathway from the pulvinar to the amygdala facilitates fear recognition. Elife. 2019;8:e40766. doi:10.7554/eLife.40766 
37. McFadyen J, Dolan RJ, Garrido MI. The influence of subcortical shortcuts on disordered sensory and cognitive processing. Nat Rev Neurosci. 2020;21(5):264-276. doi:10.1038/s41583-020-0287-1

38. McFadyen J, Mermillod M, Mattingley JB, et al. A rapid subcortical amygdala route for faces irrespective of spatial frequency and emotion. J Neurosci. 2017;37(14):3864-3874. doi:10.1523/ JNEUROSCI.3525-16.2017
39. Larner AJ. Screening utility of the Montreal Cognitive Assessment (MoCA): in place of-or as well as-the MMSE? Int Psychogeriatr. 2012;24(3):391-396. doi:10.1017/S1041610211001839

40. Cao S, Zhang J, Wang Z, et al. Laterality of attentional networks in patients with cerebral small vessel disease. Front Aging Neurosci. 2020;12:21. doi:10.3389/fnagi.2020.00021

\section{Publish your work in this journal}

Neuropsychiatric Disease and Treatment is an international, peerreviewed journal of clinical therapeutics and pharmacology focusing on concise rapid reporting of clinical or pre-clinical studies on a range of neuropsychiatric and neurological disorders. This journal is indexed on PubMed Central, the 'PsycINFO' database and CAS, and is the official journal of The International Neuropsychiatric Association (INA). The manuscript management system is completely online and includes a very quick and fair peer-review system, which is all easy to use. Visit http://www.dovepress.com/testimonials.php to read real quotes from published authors.

Submit your manuscript here: https://www.dovepress.com/neuropsychiatric-disease-and-treatment-journal 